\title{
Clinical Insights into Diffuse Alveolar Hemorrhage in Antiphospholipid Syndrome
}

\author{
Sarah Abramson Stoots ${ }^{1} \cdot$ Lindsay Lief $^{2} \cdot$ Doruk Erkan $^{3}$ \\ Published online: 6 September 2019 \\ (C) Springer Science+Business Media, LLC, part of Springer Nature 2019
}

\begin{abstract}
Purpose of Review Diffuse alveolar hemorrhage (DAH) is a rare but devastating manifestation of antiphospholipid syndrome (APS) patients with or without other systemic autoimmune diseases. Data regarding diagnosis and treatment are limited to case series. We review diagnostic and therapeutic strategies employed in APS patients with DAH and discuss our experience in managing these complex patients.

Recent Findings Pulmonary capillaritis likely contributes to the pathogenesis, however is only observed in half of the biopsies. Corticosteroids induce remission in the majority of patients, however almost half recur and require a steroid-sparing immunosuppressive to maintain remission. Cyclophosphamide- or rituximab-based regimens achieve the highest remission rates (50\%); other strategies include intravenous immunoglobulin, plasmapheresis, mycophenolate mofetil, and/or azathioprine.

Summary Given the rarity of DAH in APS, treatment is guided by interdisciplinary experience. Why certain patients achieve full remission with corticosteroids while others require immunosuppressive agents is unknown; future research should focus on the pathophysiology and optimal management.
\end{abstract}

Keywords Antiphospholipid syndrome $\cdot$ Antiphospholipid antibodies · Alveolar hemorrhage $\cdot$ Pulmonary hemorrhage · Bleeding · Capillaritis

\section{Introduction}

Antiphospholipid syndrome (APS) is a systemic autoimmune disease characterized by thrombosis and obstetric morbidity in patients with persistently elevated antiphospholipid antibodies (aPL) (lupus anticoagulant test [LA], anticardiolipin antibodies [aCL], or anti- $\beta_{2}$-glycoprotein-I antibodies [a $\left.\beta_{2} \mathrm{GPI}\right]$ ) [1]. Thrombosis in APS can be grouped as venous, arterial, or microvascular. Diffuse alveolar hemorrhage (DAH) is a rare

This article is part of the Topical Collection on Antiphospholipid Syndrome

Doruk Erkan

erkand@hss.edu

1 Department of Medicine, Weill Cornell Medicine, Hospital for Special Surgery, New York, NY, USA

2 Department of Medicine, Division of Pulmonary and Critical Care, Weill Cornell Medicine, New York, NY, USA

3 Barbara Volcker Center for Women and Rheumatic Disease, Hospital for Special Surgery, Weill Cornell Medicine, 535 E 70th Street, New York, NY 10021, USA and potentially fatal microvascular manifestation of aPL in which red blood cells (RBCs) leak from the alveolar capillaries into the intra-alveolar space [2••]. Patients present with diffuse pulmonary infiltrates, hypoxemic respiratory failure, hemoptysis, and anemia.

Diffuse alveolar hemorrhage in APS is a major therapeutic challenge, particularly in this patient population, who are hypercoagulable and often on life-long anticoagulation. Thus, the goal of this review is to offer insights into the diagnosis as well as the management of aPL-positive patients with DAH.

\section{General Considerations}

\section{How Common Is Diffuse Alveolar Hemorrhage in Antiphospholipid Syndrome?}

Diffuse alveolar hemorrhage in APS is rare. Hillerdal et al. reported the first patient in 1991 and since then, approximately 100 cases have been published [3]. In a cohort of 1000 APS patients (36\% with systemic lupus erythematosus [SLE]), pulmonary hemorrhage, adult respiratory distress syndrome 
(ARDS), and pulmonary artery thrombosis had a combined prevalence of only $0.7 \%$ [4]. One institution reported a $2 \%$ frequency of aPL-associated DAH among 483 primary APS patients followed over 16 years [5••]. In catastrophic APS (CAPS), DAH is identified in $12 \%$ of patients [6]. The nonspecific clinical and radiographic findings and inconsistent hemoptysis in DAH, as well as the requirement for an invasive diagnostic procedure, i.e., bronchoscopy, contribute to DAH being underdiagnosed [7]. Thus, the true frequency is likely higher than what is reported in the literature $[8,9]$.

\section{What Is the Etiopathogenesis of Diffuse Alveolar Hemorrhage in Antiphospholipid Syndrome?}

The defining feature of DAH is injury to the lung's microcirculation: the capillaries, arterioles, and venules lining the alveoli. Three major histologic descriptions of $\mathrm{DAH}$, not specific for APS, are (1) pulmonary capillaritis, (2) bland alveolar hemorrhage, and (3) diffuse alveolar damage [10••].

Pulmonary capillaritis is defined by neutrophilic infiltration of the perivascular interstitium (alveolar septae) of the capillaries. A vicious cycle of neutrophilic apoptosis, proteolytic enzyme disbursement, fibrin deposition, and recruitment of inflammatory cells culminates in fibrinoid necrosis of the alveolar and capillary walls. Once a critical mass of neutrophils and aforementioned debris disrupt the alveolar-capillary basement membrane, RBCs extravagate into the alveoli and interstitium. Hemosiderin-filled macrophages begin to accumulate 24-48 h after the initial vessel injury [10••, 11, 12]. Bland alveolar hemorrhage is characterized by intra-alveolar RBCs without adjacent capillary inflammation or destruction. Diffuse alveolar damage, the primary lesion in ARDS, is defined by intra-alveolar edema and hyaline membranes filled with debris lining the alveoli with sparse inflammation $[10 \bullet$, $13,14]$.

The specific mechanism behind DAH in APS is not well defined. The most frequently proposed theory is antibody-mediated pulmonary capillaritis, possibly caused by aPL-induced endothelial cell activation and upregulation of adhesion molecules [15••]. Another postulated mechanism is in situ microvascular thrombosis generating alveoli hemorrhage [16]. The detailed discussion of mechanisms of aPL-mediated DAH and micro-thrombosis can be found elsewhere [7, 15••, 17]; however, recent data suggest (a) aPL activation of the mammalian target of rapamycin (mTOR) kinase leads to endothelium proliferation and consequent vasculopathy, which is evident in aPL-associated nephropathy, and (b) complement activation is an important contributor to aPL-mediated thrombosis; however, complement deposition is rarely seen on lung biopsy [18-20].

\section{How Do Patients with Antiphospholipid Syndrome and Diffuse Alveolar Hemorrhage Present?}

Patients with DAH present with hypoxemia, hemoptysis, pleuritic chest pain, and cough. Dyspnea is the most common symptom, and fever may be present. Severity ranges from asymptomatic radiographic abnormalities to respiratory failure. Hemoptysis may be initially absent in a third of patients, because the total alveolar capacity can hold a significant pool of blood in distal airways [10••]. Acute respiratory distress syndrome may develop but is more common in CAPS [21-23].

Recurrent bleeding episodes can lead to mild to moderate (rarely severe) interstitial fibrosis and restrictive lung disease $[3,24,25]$. Development of lung hyperinflation and airflow obstruction is rare but has been reported [3, 26]. Although pulmonary hypertension in APS is primarily a result of chronic pulmonary thromboembolism and vasculopathy, DAH without embolic disease can also elicit pulmonary hypertension $[3,21,25]$.

Laboratory findings often demonstrate anemia, leukocytosis, and elevated erythrocyte sedimentation rate as well as Creactive protein; however, the absence of these findings does not exclude DAH. Imaging may show diffuse or patchy alveolar opacities. Bilateral ground glass opacities on chest computed tomography $(\mathrm{CT})$ are classically seen.

\section{Diagnosis and Evaluation of Patients Presenting With Diffuse Alveolar Hemorrhage}

The differential diagnosis for DAH includes infections, medications, systemic vasculitis, heart failure, uremia, and coagulopathies; a detailed review of differential diagnosis can be found elsewhere $[10 \bullet \cdot$.

Infection is particularly important to exclude. An opportunistic infection may present identically to an autoimmunemediated pulmonary disease but the two therapies counteract one another - the former requires a robust immune system and the latter necessitates immunosuppression [7]. Pneumonia inciting a DAH flare requires concurrent treatment [27]. The white blood count may be normal or elevated in both infectious and aPL-related hemorrhage.

Over-the-counter medications, herbal supplements, illicit drugs, toxins, and prescribed medications have been found to induce pulmonary hemorrhage. Culpable medications, (though rarely reported) include propylthiouracil, diphenylhydantoin, amiodarone, mitomycin, D-penicillamine, sirolimus, methotrexate, haloperidol, nitrofurantoin gold, all-transretinoic acid, bleomycin, montelukast, zafirlukast, inhaled cocaine, hydralazine, and infliximab [10••, 28]. Thus, it is important to review patients' medications for offending agents that may accelerate DAH in aPL-positive patients. 
During an initial presentation of DAH, a thorough work up to evaluate for other connective tissue disorders and systemic vasculitis should be undertaken. Cytoplasmic and perinuclear anti-neutrophil cytoplasmic antigens (ANCA) (anti-proteinase 3 [PR3] and anti-myeloperoxidase [MPO]) can help evaluate for granulomatosis with polyangiitis, microscopic polyangiitis, and eosinophilic granulomatosis with polyangiitis. Antinuclear antibodies (ANA), double-stranded DNA (dsDNA), complement 3 and 4 levels, urinalysis, creatinine, and anti-glomerular basement membrane antibodies (antiGBM) can help evaluate for SLE and Goodpasture's syndrome [29]; however, at times, lupus pneumonitis may be indistinguishable from DAH [30].

Cardiac echocardiogram should be obtained to exclude heart failure given the dyspnea and bilateral ground glass opacities resemble pulmonary edema, and to assess for valvular pathology. Additional imaging to exclude pulmonary embolism is often performed. Pulmonary function tests (PFTs) are rarely performed due to the difficulty patients with dyspnea have in completing the necessary maneuvers. However, if done, PFTs may show restriction; and the diffusing capacity for carbon monoxide (DLCO) may be increased due to blood in the alveolar spaces, which can absorb additional carbon dioxide [10••, 31].

Bronchoscopy with bronchoalveolar lavage (BAL) is critical in patients with APS who present with respiratory failure, dyspnea, or pulmonary infiltrates to diagnose or exclude DAH and infection. Persistent and increasing bloody returns on three sequential BAL sample aliquots support a diagnosis of DAH. Neutrophilic predominance and high numbers (greater than 20\%) of hemosiderin-laden macrophages are present on BAL cytology. Of note, increasing bloody returns may precede the hemosiderin-laden macrophages during acute hemorrhage. Yet these macrophages may persist in BAL fluid weeks to months after alveolar erythrocytes have vanished [31]. Lavage specimens should be sent for bacterial, mycobacterial, and fungal stains and cultures, fungal markers, and viral polymerase chain reaction (PCR) tests. An extensive list of infectious pathogens is reviewed elsewhere, but includes Pneumocystis jiroveci, Legionella, Mycoplasma, Nocardia, Leptospirosis, influenza A and B, respiratory syncytial virus, and adenovirus [32]. Transbronchial biopsies during bronchoscopy are not usually recommended [10••]. Surgical lung biopsy (thoracoscopic or open) is not routinely performed given the risk-benefit ratio; however, surgical biopsy may be indicated in certain circumstances when there is persistent diagnostic uncertainty.

Pathologic results of lung biopsy may reveal either pulmonary capillaritis, bland hemorrhage, or diffuse alveolar damage. Pulmonary capillaritis is particularly associated with autoimmune or inflammatory diseases; in a review of 34 lung biopsies of diffuse pulmonary hemorrhage patients (vasculitis- and connective disease-associated DAH in 14 and four cases, respectively), capillaritis was present in $88 \%$ of the specimens; however, none of these patients had APS [33]. Causes of bland alveolar hemorrhage include coagulopathies, mitral stenosis and regurgitation, congestive heart failure, and drug-induced; also, it has been reported in APS patients with DAH $[10 \bullet \cdot, 34,35]$. Diffuse alveolar damage is the classic histology observed in ARDS but also evident in infections such as Legionella, Mycoplasma, and Pneumocystis jiroveci, lymphangioleiomyomatosis, radiation therapy, bone marrow transplant complications, and APS-associated DAH [10••].

\section{What Is the Optimal Management of Diffuse Alveolar Hemorrhage in Antiphospholipid Syndrome?}

In addition to supportive care, patients are treated with highdose corticosteroids to induce remission during the acute phase of hemorrhage. Anticoagulation has not been shown to be beneficial in preventing or treating DAH in these patients. Anticoagulants are usually temporarily discontinued during the initial bleeding episode and cautiously restarted depending on the patient's clinical stability. Many patients require a steroid-sparing agent to achieve remission. Cyclophosphamide, rituximab, mycophenolate mofetil, azathioprine, plasmapheresis, and intravenous immunoglobulin (IVIG) have been used with varying degrees of success (further discussed in the "Literature Review" section). Our management approach is also described in greater detail in the "Our Experience" section.

\section{Literature Review}

\section{Methods}

We identified 91 APS cases with DAH (primary or associated with another systemic autoimmune disease, with or without catastrophic APS) through a literature review (PubMed Medline search; English only) of publications between January 1991 and December 2018 (eight case series and 15 case reports). PubMed search keywords included: "antiphospholipid," "alveolar hemorrhage," "pulmonary hemorrhage," "catastrophic antiphospholipid syndrome," "DAH," and "pulmonary capillaritis." References of prominent articles were also reviewed.

Catastrophic APS was present in 12 cases; we only included their biopsy results in the assessment of pathology findings but otherwise excluded those patients from the analysis (as this review is not focused on CAPS management) [22, 23, 36-45]. A case series of 13 patients did not report individual treatment and remission data, and thus, these patients were excluded from the outcome analysis; however, we reported their outcomes separately [25]. 
While assessing the treatment response in the remaining 66 cases, we defined "remission" or "response" as no or less than $10 \mathrm{mg}$ prednisone daily (or equivalent corticosteroid regimen) to maintain remission. For patients who received multiple immunosuppressive medications started non-simultaneously, we included each medication (excluding corticosteroids) in our analysis individually (positive response versus no response). For a small number of patients who received multiple immunosuppressive medications started simultaneously, we included the combination in our analysis (positive response versus no response, excluding corticosteroids). Because we used the most current and final information provided to gauge remission or treatment response, deceased patients were not included as treatment responders. When the data was incomplete, we exercised clinical judgment to best categorize the cases.

\section{Demographics}

Seventy-nine aPL-positive patients with DAH (excluding CAPS patients) were mostly males ( $n=59 ; 62 \%)$, often Caucasian in their fourth or fifth decade of life (mean age $48 \pm 14$ ), suggesting the possibility of a genetic disposition. Eleven patients (14\%) had SLE; however, some case series excluded patients with SLE [25, 30, 46••, 47, 48]. Eleven of 17 of patients in a small case series were current or former smokers [5••]. In a retrospective study of 63 APS patients, compared to APS patients without DAH, patients with DAH were more likely to have mitral valve disease, pulmonary hypertension, skin involvement (livedo reticularis/racemosa or chronic ulcers), obstetric morbidity, and central nervous system disease [25].

\section{Clinical Presentation}

Diffuse alveolar hemorrhage was the first presentation of APS in $9 / 79(11 \%)$ patients [15••, 21, 49-52]; three out of 17 patients in one case series were diagnosed with APS only after they presented with DAH [5••]. Yet, two other reviews of 18 and 13 patients noted a median DAH onset of 5.9 and 5.8 years, respectively, after the diagnosis of APS [25, 46]. Furthermore, many patients have a delay in diagnosis [53, 54], e.g., a 26-year-old female visited her primary care doctor and emergency room for multiple episodes of hemoptysis throughout 1 year before the eventual diagnosis of APS [49].

\section{Triggers}

Viral upper respiratory tract infections or bacterial pneumonia were both reported to incite episodes of alveolar hemorrhage $[15 \bullet \bullet, 27]$. While periods of supratherapeutic international normalized ratio (INR) could pre-dispose patients to alveolar hemorrhage, some patients had alveolar hemorrhage while off anticoagulation, and the vast majority of published cases revealed sub-therapeutic or therapeutic INR on presentation. Four patients did develop recurrent DAH with a supratherapeutic INR and one patient's first episode of DAH began 3 weeks after starting warfarin [15••, 25, 27, 46]. Half of patients (52\%) were on anticoagulation (typically warfarin) either before or after the diagnosis of DAH, but true prevalence of anticoagulation use is likely higher than reported given the limitations of these studies.

\section{Pathology}

Pulmonary capillaritis was seen more frequently than microvascular thrombosis. Half of all published cases with a tissue biopsy

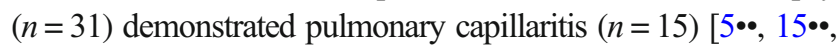
24, 25, 38, 41, 46••, 53, 55-57] (Table 1). Micro-thrombosis was present in only seven of 31 biopsies (23\%), and five of the seven concomitantly displayed capillaritis [24, 25, 41, 46••, 54, 56, 57]. Interestingly, despite extensive microvascular thrombosis on biopsy, large vessel pulmonary emboli were absent in all seven patients. Bland alveolar hemorrhage or diffuse alveolar damage with or without the presence of mild interstitial inflammation was seen in $45 \%$ of biopsies [3, 5••, 25, 30, 36, 45, 46••, 50, 58].

\section{Outcomes (Remission, Recurrence, and Death)}

Across the 66 cases included in our outcomes analysis, 43 patients $(65 \%)$ ultimately achieved remission, 19/43 (44\%) with corticosteroids or supportive care alone (Fig. 1), and 24/ $43(55 \%)$ with immunosuppressive agent(s). At one institution, $10(59 \%)$ of 17 patients achieved complete and sustained remission; interestingly, four of these 10 patients had a mild form of DAH and reached remission without even corticosteroid treatment (details not reported). In the same case series, three $(18 \%)$ patients relapsed within a median time of 9 months [5*0]. Another large case series $(n=18)$ demonstrated poorer remission rates: $39 \%$ achieved complete remission and $28 \%$ were alive but remained on high-dose corticosteroids after 1 to 2 years follow up; $72 \%$ of all patients experienced recurrent disease [46••].

Based on our review, 36 of 66 patients (55\%) experienced recurrent disease, which may underestimate the true relapse rate, since many groups did not report long-term follow up (mean follow up was 35 months; however follow up data was not provided for 11 patients). Symptoms often reappeared during corticosteroid tapering, and many patients were never able to discontinue prednisone.

We found the mortality rate among all cases as $21 \%$. This was similar to published rates in patients with DAH secondary to numerous etiologies: $25 \%$ during an acute episode and $16 \%$ among those who survived their initial hospitalization [59]. Reported mortality in SLE-associated DAH has ranged widely from $12 \%$ in two studies up to $36 \%, 42 \%$, and $68 \%$ in others [60-64]. Data were limited but similar in primary 
Table 1 Pathology of $31^{\text {a }}$ lung biopsies in antiphospholipid syndrome patients with diffuse alveolar hemorrhage

\begin{tabular}{lr} 
Pathology & $N(\%)$ \\
\hline Pulmonary capillaritis & $10(32 \%)$ \\
$\begin{array}{ll}\text { Microvascular thrombosis } \\
\text { Both pulmonary capillaritis and microvascular thrombosis }\end{array}$ & $2(6 \%)$ \\
Bland alveolar hemorrhage with/without nonspecific/interstitial inflammation, or diffuse alveolar damage (no capillaritis or thrombosis) & $5(16 \%)$ \\
\hline
\end{tabular}

${ }^{\text {a }}$ Only 31 of the 91 reported cases underwent lung biopsy

${ }^{\mathrm{b}}$ Findings suggestive of small vessel vasculopathy (myointimal thickening/remodeling, thickened arteriole walls, and increased fibroblasts) were mentioned in three biopsies [46••, 54, 57]

APS, however ranged between 23 and $33 \%$ [5••, 25, 46••]. Cause of death was often described as due to "uncontrolled DAH"; however, massive hemorrhage was only rarely described [46••]. Documented causes of death included sepsis, respiratory failure due to pulmonary infarction and pneumonia, stroke, spontaneous intracranial hemorrhage, and pulmo-

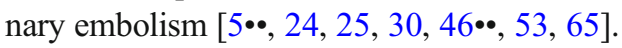

\section{Treatment}

Table 2 illustrates major therapeutic interventions utilized and subsequent clinical response. In general, cyclophosphamide- or rituximab-based regimens achieved the highest remission rates (approximately 50\%), which aligns with the largest case series reported to date [46••]. Intravenous immunoglobulin (IVIG) and plasma exchange together with other immunosuppressive agents demonstrated similar treatment responses (approximately 30\%), whereas mycophenolate mofetil and azathioprine use resulted in lower remission rates (approximately 15\%).
Given the efficacy of cyclophosphamide in granulomatosis with polyangiitis and other pulmonary vasculitides, this medication was generally utilized initially in the management of aPL-associated DAH [31]. Patients unable to achieve remission with cyclophosphamide ultimately died, remained on high-dose corticosteroids, or responded to rituximab or IVIG [15••, 24, 27, 46••, 66].

Recent papers have shown success with rituximab, an antiCD20 monoclonal antibody that targets a surface protein expressed on B-lymphocytes, the precursor of aPLproducing plasma cells. For instance, the first patient with primary APS treated with rituximab was a 32-year-old male who had been hospitalized 15 times over 18 months for recurrent DAH; after unsuccessful treatment with azathioprine, IVIG, cyclophosphamide, and plasmapheresis, two doses of rituximab ( $1 \mathrm{~g}$ each 2 weeks apart) led to a sustained remission over 2 years of follow up [66]. Others have reported similar success with follow up ranging from 1 to 8 years [46••, 47].

Several authors have achieved remission using IVIG, both acutely to control an active disease flare and long-term with monthly maintenance infusions. For instance, Janowiak et al.
Fig. 1 Outcomes in diffuse alveolar hemorrhage. ${ }^{1}$ Includes two patients whom initially achieved remission but then flared without further remission. ${ }^{2}$ Includes three patients whom achieved full or partial remission with cyclophosphamide but later died of (1) sepsis, (2) bone marrow transplantation complications, and (3) diffuse alveolar hemorrhage flare when cyclophosphamide was discontinued [46••, 53]

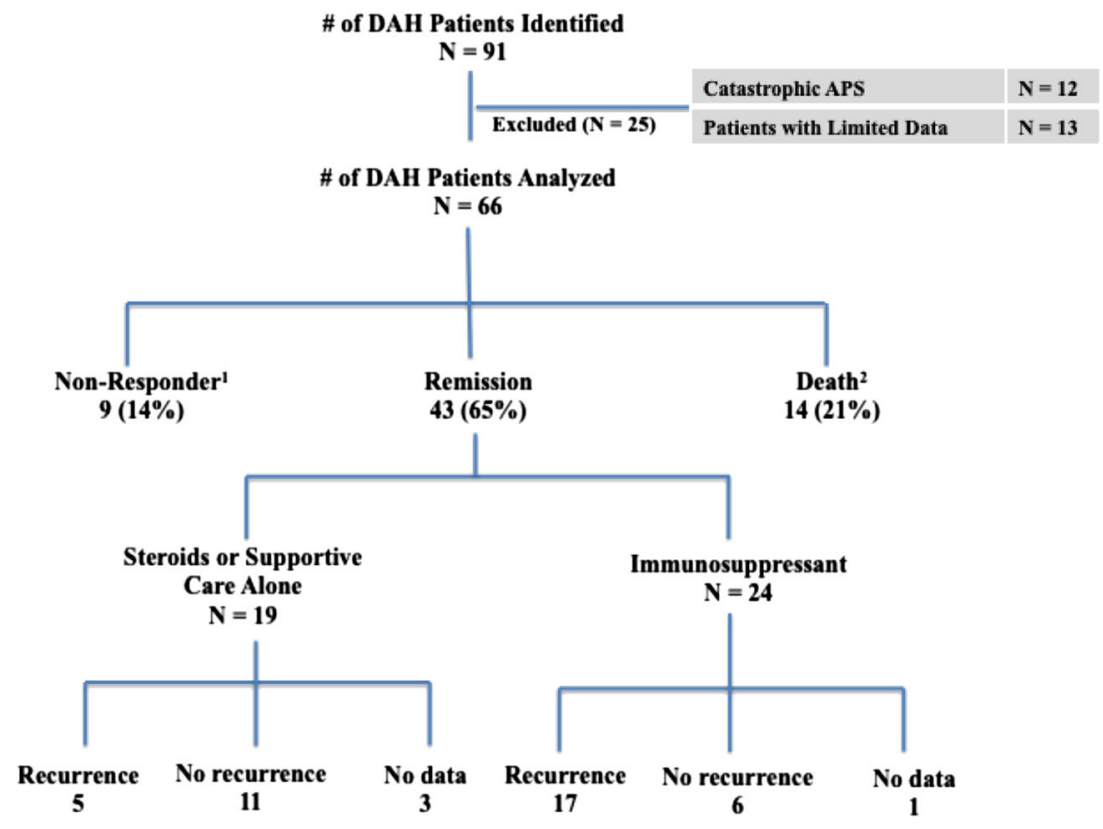


Table 2 Treatment patterns and response in antiphospholipid syndrome patients with diffuse alveolar hemorrhage ${ }^{\mathrm{a}}$

\begin{tabular}{lccl}
\hline Treatment & Number & Response $^{\mathrm{b}}$ & Mean no. of failed and/or concurrent medications required before response $^{\mathrm{c}}$ \\
\hline No immunosuppression $^{\mathrm{d}}$ & 5 & $4(80 \%)$ & - \\
Corticosteroid only & 20 & $15(75 \%)$ & - \\
Cyclophosphamide (CYC) & 22 & $11(50 \%)$ & 0.9 \\
Rituximab (RTX) & 10 & $4(40 \%)$ & 2.8 \\
Intravenous Immunoglobulin & 11 & $4(36 \%)$ & 1.8 \\
Mycophenolate mofetil (MMF) & 13 & $2(15 \%)$ & 2.0 \\
Azathioprine (AZA) & 14 & $2(14 \%)$ & 0.5 \\
Plasmapheresis + CYC, MMF, or AZA & 11 & $4(36 \%)$ & 2.0 \\
CYC+ methotrexate & 1 & $1(100 \%)$ & 0 \\
CYC + RTX & 1 & $1(100 \%)$ & 3 \\
RTX + MMF & 2 & $2(100 \%)$ & 0.5 \\
\hline
\end{tabular}

${ }^{a}$ Excluding 13 patients from one case series where individual outcome data was not reported. Of 72 diffuse alveolar hemorrhage episodes in these 13 patients, no immunosuppression was given in $25(35 \%)$ episodes, steroids were given in $47(65 \%)$ episodes, and secondary immunosuppression (azathioprine, cyclophosphamide, IVIG, plasmapheresis, and rituximab) was used in three patients with recurrent disease [25]

b Please refer to the text ("Literature Review," "Methods" section) for the definition of "response"

${ }^{\mathrm{c}}$ Mean number of previous or concurrent immunosuppressive medications failed (excluding corticosteroids) at the time of "response." Of note, all patients treated with immunosuppressive medications initially received steroids

${ }^{\mathrm{d}}$ Four patients in one case series achieved remission without immunosuppression (including corticosteroids); details not reported except the mean follow up of 69 months [5••]

described a patient with six recurrent DAH episodes and multiple infections who only remained disease-free after the addition of monthly IVIG to mycophenolate and prednisone [27]. Others report similar success with IVIG $[15,51]$; however, some patients did not achieve remission with IVIG [46••, 65, 66]. Plasma exchange, which is traditionally used in CAPS, has been effective in a third of patients with DAH without CAPS when used in combination with other immunosuppressive agents [5••, 50, 57, 67, 68].

Monotherapy with mycophenolate mofetil had a relatively low response rate $[15 \bullet \cdot, 27,46 \bullet \bullet, 47,68]$; however, the combination of rituximab and mycophenolate mofetil resulted in complete remission in two patients [46••]. None of the six patients on monotherapy azathioprine in the largest case series attained remission [46*•], and others reported similar outcomes $[25,30,50,53]$.

Hydroxychloroquine was used as monotherapy in only two cases (one resulted in remission and the other treatment failure) and demonstrated intermittent success when utilized as an adjunct agent [5••, 46••, 47, 66, 68].

\section{Our Experience}

\section{Diagnostic Considerations}

In patients with clinically significant aPL profiles (persistently positive LA test and/or moderate-to-high titer aCL/a $\beta_{2} \mathrm{GPI}$ $\mathrm{IgG} / \mathrm{M})$, when imaging studies are suggestive of $\mathrm{DAH}$, bronchoscopy is important during initial or ambiguous presentations. During the initial diagnostic assessment, we also obtain chest $\mathrm{CT}$ with contrast as pulmonary embolism can present with hemoptysis. In patients with APS and recurrent, welldocumented DAH and without suspicion for additional underlying disease or infection, we generally do not perform repeat bronchoscopy.

We do not routinely recommend lung biopsy due to associated risks in patients who are generally anticoagulated and at high risk for thrombosis. Lung biopsy is considered if the etiology of the DAH is unclear from the serologic work up and an accurate diagnosis cannot otherwise be made. If performed, the pathologist should perform immunofluorescent staining to assess for immune-complex and complement deposition, and tissue cultures should be sent as reviewed above. Of note, if the clinical scenario and laboratory tests suggest APS, the absence of capillaritis or microthrombosis on biopsy should not preclude the APS diagnosis.

Minor hemoptysis presents a diagnostic dilemma when it develops in a patient with APS on warfarin. Is the warfarin to blame? We urge providers to consider DAH on the differential to avoid missed and delayed diagnoses. And in those with documented APS, high clinical suspicion for DAH is necessary, as documented cases of hemoptysis incorrectly attributed to warfarin overdose rather than a first occurrence of alveolar hemorrhage have been reported [27]. In addition, when patients do not yet carry the DAH diagnosis, symptoms may be falsely attributed to respiratory infections. 


\section{Therapeutic Considerations}

Given the rarity of the disease, lack of controlled studies, limited literature regarding treatment, and heterogeneity, there is no "one size fits all" approach to treatment. In our experience, two different presentations of aPL-positive patients with DAH exist: (a) acute - with moderate to severe pulmonary hemorrhage (with varying degrees of respiratory failure) requiring hospitalization and (b) chronic - with mild hemoptysis, dyspnea, and/or positive imaging findings, usually seen as an outpatient. Patients in both settings often require steroids for disease control and a steroid-sparing immunosuppressive for maintenance therapy, as we discuss below.

In hospitalized patients, supportive care is a mainstay of acute management, ranging from supplemental oxygen to mechanical ventilation, along with as-needed transfusions of blood products. Empiric antibiotic therapy is often coadministered with corticosteroids at initial presentation and subsequently if a patient clinically decompensates. Of note, immunosuppressed APS patients with DAH are especially susceptible to pulmonary infections (typically bacterial), which can manifest with hemoptysis. We do not routinely treat with antiviral or antifungal antibiotics unless laboratory or culture data suggest a particular pathogen. High-dose corticosteroids (intravenous methylprednisolone 500-1000 mg daily for 3 days, followed by oral prednisone $1 \mathrm{mg} / \mathrm{kg}$ daily in divided doses (maximum $60 \mathrm{mg}$ day)) are generally the primary treatment during the acute phase. Due to high mortality in these patients, corticosteroids are rapidly initiated even when diagnostic tests are still pending. For patients with life threatening or ventilator-dependent DAH, usually in the setting of CAPS, we also use plasma exchange and/or intravenous immunoglobulin [69].

Anticoagulation therapy is temporarily discontinued during the acute severe bleeding episode; however, the risks and benefits of stopping anticoagulation should be carefully calculated in aPL-positive patients with/without history of thrombosis. When stopped, we recommend restarting anticoagulation as soon as the acute bleeding is controlled. In cases with concurrent pulmonary embolism and hemorrhage, starting anticoagulation and corticosteroids simultaneously may be required [70]. A multidisciplinary team approach should appraise the risks and benefits, i.e., timing and dosage of safe anticoagulation [71]. In aPL-positive patients without a history of thrombosis presenting with DAH, anticoagulation does not treat or prevent DAH and we do not recommend anticoagulation for treatment purposes. However, we recommend continuing prophylactic dose anticoagulation to prevent thrombosis in hospitalized patients.

In an outpatient setting with mild DAH in the absence of respiratory compromise, we recommend initiating oral prednisone (or increasing the dose to) $1 \mathrm{mg} / \mathrm{kg}$ daily to a maximum of $60 \mathrm{mg}$ daily. As discussed above, in patients with suspected new-onset DAH, urgent referral to a pulmonary team for confirmatory bronchoscopy is required. For patients with known DAH and recurrent albeit mild symptoms, oral corticosteroids with close follow up is often sufficient.

Both in an inpatient and outpatient setting, in addition to steroids, we recommend starting steroid-sparing immunosuppressive agents as, based on our experience, the risk of recurrence is relatively high during corticosteroid tapering. Our first-line therapy is generally mycophenolate mofetil (quickly accelerating the dose to $3 \mathrm{~g} /$ day) with or without rituximab (1000 mg twice 2 weeks apart), once infectious etiologies have been excluded. We add rituximab to mycophenolate mofetil especially in aPL-positive patients with hematologic and/ or other microthrombotic manifestations, e.g., thrombocytopenia or livedoid vasculopathy, and also in patients hospitalized with relatively severe hemorrhage. After the DAH is controlled, we decrease the prednisone dose with close monitoring, usually by $10-15 \mathrm{mg}$ per month. We do not use cyclophosphamide as first-line therapy due to the high incidence of treatment-associated toxicity.

In cases of recurrent DAH on mycophenolate mofetil, we add rituximab depending on the other aPL-manifestations discussed above. No data exist on the long-term management of rituximab in APS patients with DAH; we generally do not continue rituximab if there is full recovery. If patients have no response or recurrence of DAH despite the combination of mycophenolate mofetil and rituximab, we next add IVIG. We have treated several patients who achieved remission with the addition of IVIG $2 \mathrm{~g} / \mathrm{kg}$ over $4-5$ days, every 1-3 months, to mycophenolate mofetil (with or without a history of rituximab use) (unpublished data).

In all aPL-positive patients with DAH, despite the lack of clinical data, we add hydroxychloroquine (200-400 mg daily) and a statin (e.g., atorvastatin $10-20 \mathrm{mg}$ /day) to the treatment regimen. Hydroxychloroquine reduces the risk of thrombosis in experimental aPL/APS models and lupus patients; similarly, statins ameliorate the proinflammatory and prothrombotic markers in aPL-positive patients [72-75].

\section{Prognostic Considerations}

We do not understand why certain patients experience a single episode that clears with corticosteroids, while others require long-term immunosuppressive agents. Based on our experience, patients may continue to have abnormalities on imaging despite resolution of symptoms. Some of these patients, whose symptoms of DAH have resolved, but have persistent opacities on imaging, may have recurrence of mild hemoptysis during upper respiratory tract infections or when their INR is supratherapeutic. In asymptomatic patients with persistent imaging findings, we continue the immunosuppressive agents while tapering and ultimately discontinuing prednisone as discussed above. 
Monitoring for recurrence can be challenging, particularly when patients are asymptomatic with normal laboratory findings. For patients on chronic immunosuppression for prior hemorrhagic episodes, we recommend obtaining chest radiograph and pulmonary function tests including DLCO yearly or with changes in respiratory symptoms.

In our experience, patients with DAH (without CAPS) have lower mortality rates than those reported in the literature; we attribute this to publication bias for this rare condition.

\section{Conclusion}

Diffuse alveolar hemorrhage is a rare yet potentially fatal complication of APS, in which red blood cells leak from the alveolar capillaries into the intra-alveolar space. Pulmonary capillaritis, along with antibody-mediated endothelial proliferation, likely contributes to its pathogenesis, yet is not always observed pathologically. Diffuse alveolar hemorrhage typically presents with hypoxemia and subsequent respiratory failure, anemia, hemoptysis, and diffuse radiographic pulmonary infiltrates.

Given the rarity of pulmonary hemorrhage in APS, no randomized trials exist. Treatment is guided by case reports, case series, and inter-disciplinary experience. While anticoagulation is standard of care for the treatment and prevention of thrombotic complications of APS, its use is contraindicated during acute DAH episodes and is not efficacious in preventing DAH. In addition to supportive care, high-dose corticosteroids are the initial treatment for DAH in APS. The majority of patients require an immunosuppressive steroid sparing agent; we generally use mycophenolate mofetil with or without rituximab as a first-line treatment strategy, and add IVIG, plasmapheresis, and cyclophosphamide in patients with limited response.

\section{References}

Papers of particular interest, published recently, have been highlighted as:

•• Of major importance

1. Miyakis S, Lockshin MD, Atsumi T, Branch DW, Brey RL, Cervera $\mathrm{R}$, et al. International consensus statement on an update of the classification criteria for definite antiphospholipid syndrome (APS). J Thromb Haemost. 2006;4(2):295-306. https://doi.org/10. 1111/j.1538-7836.2006.01753.x.

2.• Garcia D, Erkan D. Diagnosis and management of the antiphospholipid syndrome. N Engl J Med. 2018;378(21):2010 21. https://doi.org/10.1056/NEJMra1705454 Clear and concise overview of APS pathogenesis.
3. Hillerdal G, Hägg A, Licke G, Wegenius G, Scheibenpflug L. Intraalveolar haemorrhage in the anticardiolipin antibody syndrome. Scand J Rheumatol. 1991;20(1):58-62. https://doi.org/10.3109/ 03009749109165923.

4. Cervera R, Piette JC, Font J, Khamashta MA, Shoenfeld Y, Camps MT, et al. Antiphospholipid syndrome: clinical and immunologic manifestations and patterns of disease expression in a cohort of 1 , 000 patients. Arthritis Rheum. 2002;46(4):1019-27. https://doi.org/ 10.1002/art.10187.

5.• Yachoui R, Sehgal R, Amlani B, Goldberg JW. Antiphospholipid antibodies-associated diffuse alveolar hemorrhage. Semin Arthritis Rheum. 2015;44(6):652-7. https://doi.org/10.1016/j.semarthrit. 2014.10.013 One of the two largest and recent case series with a thorough overview of DAH presentation.

6. Rodriguez-Pinto I, Moitinho M, Santacreu I, Shoenfeld Y, Erkan D, Espinosa G, et al. Catastrophic antiphospholipid syndrome (CAPS): descriptive analysis of 500 patients from the International CAPS Registry. Autoimmun Rev. 2016;15(12): 1120-4. https://doi.org/10.1016/j.autrev.2016.09.010.

7. Asherson RA, Cervera R, Shepshelovich D, Shoenfeld Y. Nonthrombotic manifestations of antiphospholipid syndrome: away from thrombosis? J Rheumatol. 2006;33(6):1038-44.

8. Asherson RA, Cervera R, Piette JC, Font J, Lie JT, Burcoglu A, et al. Catastrophic antiphospholipid syndrome. Clinical and laboratory features of 50 patients. Medicine. 1998;77(3):195-207. https:// doi.org/10.1097/00005792-199805000-00005.

9. Cervera R, Espinosa RG. Unusual manifestations of the antiphospholipid syndrome. Int J Clin Rheumatol. 2009;4(2): 189-202. https://doi.org/10.2217/ijr.09.3.

10.• Ioachimescu OC, Stoller JK. Diffuse alveolar hemorrhage: diagnosing it and finding the cause. Cleveland Clinical Journal of Medicine. 2008;75(4):258-80. https://doi.org/10.3949/ccjm.75.4.258 Excellent review of the differential diagnosis of DAH.

11. Colby TV, Fukuoka J, Ewaskow SP, Helmers R, Leslie KO. Pathologic approach to pulmonary hemorrhage. Ann Diagn Pathol. 2001;5(5):309-19. https://doi.org/10.1053/adpa.2001. 27923.

12. Lara AR, Schwarz MI. Diffuse alveolar hemorrhage. Chest. 2010;137(5):1164-71. https://doi.org/10.1378/chest.08-2084.

13. Beasley MB. The pathologist's approach to acute lung injury. Arch Pathol Lab Med. 2010;134(5):719-27. https://doi.org/10.1043/ 1543-2165-134.5.719.

14. Parambil JG, Myers JL, Aubry MC, Ryu JH. Causes and prognosis of diffuse alveolar damage diagnosed on surgical lung biopsy. Chest. 2007;132(1):50-7. https://doi.org/10.1378/chest.07-0104.

15.•- Deane KD, West SG. Antiphospholipid antibodies as a cause of pulmonary capillaritis and diffuse alveolar hemorrhage: a case series and literature review. Semin Arthritis Rheum. 2005;35(3):154 65. https://doi.org/10.1016/j.semarthrit.2005.05.006 Excellent discussion of proposed mechanisms of DAH and pulmonary capillaritis.

16. Malik AB. Mechanisms of lung vascular injury and edema induced by pulmonary microembolism. Med Principles Pract. 1990;2(1):19. https://doi.org/10.1159/000157326.

17. Asherson RA, Cervera R, Wells AU. Diffuse alveolar hemorrhage: a nonthrombotic antiphospholipid lung syndrome? Semin Arthritis Rheum. 2005;35(3):138-42. https://doi.org/10.1016/j.semarthrit. 2005.08.006.

18. Girardi G, Berman J, Redecha P, Spruce L, Thurman J, Kraus D, et al. Complement $\mathrm{C} 5$ a receptors and neutrophils mediate fetal injury in the antiphospholipid syndrome. J Clin Invest. 2003;112(11): 1644-54. https://doi.org/10.1172/JCI200318817.

19. Pierangeli SS, Girardi G, Vega-Ostertag M, Liu X, Espinola RG, Salmon J. Requirement of activation of complement C3 and C5 for antiphospholipid antibody-mediated thrombophilia. Arthritis Rheum. 2005;52(7):2120-4. https://doi.org/10.1002/art.21157. 
20. Canaud G, Bienaime F, Tabarin F, Bataillon G, Seilhean D, Noel $\mathrm{LH}$, et al. Inhibition of the mTORC pathway in the antiphospholipid syndrome. N Engl J Med. 2014;371(4):303-12. https://doi.org/10. 1056/NEJMoa1312890.

21. Espinosa RG, Cervera R, Font J, Asherson RA. The lung in the antiphospholipid syndrome. Ann Rheum Dis. 2002;61(3):195-8. https://doi.org/10.1136/ard.61.3.195.

22. Camera A, Rocco S, De Lucia D, Costantini S, Lucania A, Servillo $\mathrm{G}$, et al. Reversible adult respiratory distress in primary antiphospholipid syndrome. Haematologica. 2000;85(2):208-10.

23. Makhoul BF, Guralnik L, Azzam ZS. Catastrophic antiphospholipid syndrome presented with abdominal, pulmonary, and bone marrow complications. Rheumatol Int. 2010;30(3):4014. https://doi.org/10.1007/s00296-009-0946-z.

24. Kerr JE, Poe R, Kramer Z. Antiphospholipid antibody syndrome presenting as a refractory noninflammatory pulmonary vasculopathy. Chest. 1997;112(6):1701-10. https://doi.org/10.1378/chest. 112.6.1707.

25. Scheiman Elazary A, Cohen MJ, Aamar S, Dranitzki Z, TayerShifman O, Mevorach D, et al. Pulmonary hemorrhage in antiphospholipid antibody syndrome. J Rheumatol. 2012;39(8): 1628-31. https://doi.org/10.3899/jrheum. 120205.

26. Schwarz MI, Mortenson RL, Colby TV, Waldron JA, Lynch DA, Hutt MP, et al. Pulmonary capillaritis. The association with progressive irreversible airflow limitation and hyperinflation. Am Rev Respir Dis. 1993;148(2):507-11. https://doi.org/10.1164/ajrccm/ 148.2.507.

27. Janowiak P, Sieminska A, Porzezinska M, Smolenska Z, Suchanek $\mathrm{H}$, Jassem E. Difficulties in the treatment of recurring diffuse alveolar hemorrhage accompanying primary antiphospholipid syndrome: a case report and literature review. Adv Respir Med. 2018;86(3):126-30. https://doi.org/10.5603/ARM.2018.0020.

28. Paley MA, Edrees F, Kudose S, Gaut JP, Ranganathan P, Vijayan A. Successful use of rituximab for hydralazine-induced anti-neutrophil cytoplasmic antibodies-associated vasculitis. Saudi J Kidney Dis Transpl. 2019;30(1):226-30. https://doi.org/10.4103/1319-2442. 252915.

29. Gerosa M, Meroni PL, Erkan D. Recognition and management of antiphospholipid syndrome. Curr Opin Rheumatol. 2016;28(1):519. https://doi.org/10.1097/BOR.0000000000000240.

30. Nguyen VA, Gotwald T, Prior C, Obermoser G, Sepp N. Acute pulmonary edema, capillaritis and alveolar hemorrhage: pulmonary manifestations coexistent in antiphospholipid syndrome and systemic lupus erythematosus? Lupus. 2005;14(7):557-60. https:// doi.org/10.1191/0961203305lu2107cr.

31. Krause ML, Cartin-Ceba R, Specks U, Peikert T. Update on diffuse alveolar hemorrhage and pulmonary vasculitis. Immunol Allergy Clin N Am. 2012;32(4):587-600. https://doi.org/10.1016/j.iac. 2012.08.001

32. von Ranke FM, Zanetti G, Hochhegger B, Marchiori E. Infectious diseases causing diffuse alveolar hemorrhage in immunocompetent patients: a state-of-the-art review. Lung. 2013;191(1):9-18. https:// doi.org/10.1007/s00408-012-9431-7.

33. Travis WD, Colby TV, Lombard C, Carpenter HA. A clinicopathologic study of 34 cases of diffuse pulmonary hemorrhage with lung biopsy confirmation. Am J Surg Pathol. 1990;14(12):111225. https://doi.org/10.1097/00000478-199012000-00003.

34. Jonckheer J, Slabbynck H, Spapen H. Diffuse alveolar haemorrhage with predominant upper lung lobe involvement associated with congestive heart failure: a case series. J Thorac Dis. 2017;9(10):3956-60. https://doi.org/10.21037/jtd.2017.09.92.

35. Tamai K, Tomii K, Nakagawa A, Otsuka K, Nagata K. Diffuse alveolar hemorrhage with predominantly right-sided infiltration resulting from cardiac comorbidities. Intern Med. 2015;54(3): 319-24. https://doi.org/10.2169/internalmedicine.54.3057.
36. Ghosh S, Walters HD, Joist JH, Osborn TG, Moore TL. Adult respiratory distress syndrome associated with antiphospholipid syndrome. J Rheumatol. 1993;20(8):1406-8.

37. Iglesias-Jimenez E, Camacho-Lovillo M, Falcon-Neyra D, LirolaCruz J, Neth O. Infant with probable catastrophic antiphospholipid syndrome successfully managed with rituximab. Pediatrics. 2010;125(6):e1523-8. https://doi.org/10.1542/peds.2009-2939.

38. Kazzaz NM, Wilson AM, Kado R, Barnes GD, Knight JS. A 37year-old man with primary antiphospholipid syndrome presenting with respiratory distress and worsening toe ischemia. Arthritis Care Res. 2017;69(8):1253-9. https://doi.org/10.1002/acr.23168.

39. Kronbichler A, Frank R, Kirschfink M, Szilagyi A, Csuka D, Prohaszka Z, et al. Efficacy of eculizumab in a patient with immunoadsorption-dependent catastrophic antiphospholipid syndrome: a case report. Medicine (Baltimore). 2014;93(26):e143. https://doi.org/10.1097/MD.0000000000000143.

40. Martis N, Blanchouin E, Lazdunski R, Lechtman S, Robert A, Hyvernat $\mathrm{H}$, et al. A therapeutic challenge: catastrophic antiphospholipid syndrome with diffuse alveolar haemorrhage. Immunol Res. 2015;62(2):222-4. https://doi.org/10.1007/s12026015-8649-x.

41. Pieralli F, Grazzini M, Vannucchi V, Mancini A, Cammelli D, Nozzoli C. Recurrent episodes of hemorrhagic alveolitis in relapsing catastrophic antiphospholipid syndrome: the same side of the dark moon. Clin Rheumatol. 2014;33(3):429-33. https://doi.org/ 10.1007/s10067-013-2454-5.

42. Rangel ML, Alghamdi I, Contreras G, Harrington T, Thomas DB, Barisoni L, et al. Catastrophic antiphospholipid syndrome with concurrent thrombotic and hemorrhagic manifestations. Lupus. 2013;22:855-64. https://doi.org/10.1177/0961203313491024.

43. Vieregge GB, Harrington TJ, Andrews DM, Carpintero MF, Green DF, Nayer A. Catastrophic antiphospholipid syndrome with severe acute thrombotic microangiopathy and hemorrhagic complications. Case Rep Med. 2013;2013:915309-6. https://doi.org/10.1155/ 2013/915309.

44. Wan T, Tsang P. Catastrophic antiphospholipid syndrome presenting with pulmonary hemorrhage: case report. J Thromb Thrombolysis. 2015;39(1):68-70. https://doi.org/10.1007/s11239014-1089-y.

45. Wiedermann FJ, Mayr A, Schobersberger W, Knotzer H, Sepp N, Rieger M, et al. Acute respiratory failure associated with catastrophic antiphospholipid syndrome. J Intern Med. 2000;247(6):723-30. https://doi.org/10.1046/j.1365-2796.2000.00687.x.

46.• Cartin-Ceba R, Peikert T, Ashrani A, Keogh K, Wylam ME, Ytterberg S, et al. Primary antiphospholipid syndrome-associated diffuse alveolar hemorrhage. Arthritis Care Res. 2014;66(2):30110. https://doi.org/10.1002/acr.22109 The largest case series of APS patients with DAH accompanied by a literature review.

47. Aakjaer S, Bendstrup E, Ivarsen P, Madsen LB. Continous rituximab treatment for recurrent diffuse alveolar hemorrhage in a patient with systemic lupus erythematosus and antiphosholipid syndrome. Respir Med Case Rep. 2017;22:263-5. https://doi.org/10.1016/j. rmcr.2017.09.012

48. Hambly N, Sekhon S, McIvor RA. Antiphospholipid antibody syndrome: diffuse alveolar hemorrhage and libman-sacks endocarditis in the absence of prior thrombotic events. Ulster Med J. 2014;83(1): 47-57.

49. Bielski B, Shah NH, Westerveld D, Stalvey C. Diffuse alveolar haemorrhage and Libman-Sacks endocarditis: a rare presentation of antiphospholipid syndrome. BMJ Case Rep. 2018;10. https:// doi.org/10.1136/bcr-2017-223728.

50. Koolaee RM, Moran AM, Shahane A. Diffuse alveolar hemorrhage and Libman-Sacks endocarditis as a manifestation of possible primary antiphospholipid syndrome. J Clin Rheumatol. 2013;19(2): 79-83. https://doi.org/10.1097/RHU.0b013e318278c577. 
51. Gertner E. Diffuse alveolar hemorrhage in the antiphospholipid syndrome: spectrum of disease and treatment. J Rheumatol. 1999;26(4):805-7.

52. Hyde R, Runnstrom M, Anderson A, Riley L, Ataya A. Primary antiphospholipid syndrome associated LiebmanSachs endocarditis leading to diffuse alveolar hemorrhage: a case report. Respir Med Case Rep. 2018;25:181-3. https://doi. org/10.1016/j.rmcr.2018.08.018.

53. Asherson RA, Greenblatt MA. Recurrent alveolar hemorrhage and pulmonary capillaritis in the "primary" antiphospholipid syndrome. J Clin Rheumatol. 2001;7(1):30-3. https://doi.org/10.1097/ 00124743-200102000-00007.

54. Maggiorini M, Knoblauch A, Schneider J, Russi EW. Diffuse microvascular pulmonary thrombosis associated with primary antiphospholipid antibody syndrome. Eur Respir J. 1997;10:72730. https://doi.org/10.1183/09031936.97.10030727.

55. Crausman RS, Achenbach GA, Pluss WT, O'Brien RF, Jennings CA. Pulmonary capillaritis and alveolar hemorrhage associated with the antiphospholipid antibody syndrome. J Rheumatol. 1996;22(3):554-6.

56. Gertner E, Lie JT. Pulmonary capillaritis, alveolar hemorrhage, and recurrent microvascular thrombosis in primary antiphospholipid syndrome. J Rheumatol. 1993;20(7):1224-8.

57. Waterer GW, Latham B, Waring JA, Gabbay E. Pulmonary capillaritis associated with the antiphospholipid antibody syndrome and rapid response to plasmapheresis. Respirology. 1999;4(4):4058. https://doi.org/10.1046/j.1440-1843.1999.00212.x.

58. Savin H, Huberman M, Kott E, Lishner M, Kitai Y, Kidron D, et al. Fibrosing alveolitis associated with primary antiphospholipid syndrome. Br J Rheumatol. 1994;33(10):977-80. https://doi.org/10. 1093/rheumatology/33.10.977.

59. de Prost N, Parrot A, Picard C, Ancel PY, Mayaud C, Fartoukh M, et al. Diffuse alveolar haemorrhage: factors associated with inhospital and long-term mortality. Eur Respir J. 2010;35(6):130311. https://doi.org/10.1183/09031936.00075309.

60. Badsha H, Teh CL, Kong KO, Lian TY, Chng HH. Pulmonary hemorrhage in systemic lupus erythematosus. Semin Arthritis Rheum. 2004;33(6):414-21. https://doi.org/10.1016/j.semarthrit. 2003.09.006

61. Canas C, Tobon GJ, Granados M, Fernandez L. Diffuse alveolar hemorrhage in Colombian patients with systemic lupus erythematosus. Clin Rheumatol. 2007;26(11):1947-9. https://doi.org/10. 1007/s10067-007-0576-3.

62. Martinez-Martinez MU, Abud-Mendoza C. Predictors of mortality in diffuse alveolar haemorrhage associated with systemic lupus erythematosus. Lupus. 2011;20(6):568-74. https://doi.org/10.1177/ 0961203310392430.

63. Santos-Ocampo AS, Mandell BF, Fessler BJ. Alveolar hemorrhage in systemic lupus erythematosus. Chest. 2000;118(4):1083-90. https://doi.org/10.1378/chest.118.4.1083.

64. Zamora MR, Warner ML, Tuder R, Schwarz MI. Diffuse alveolar hemorrhage and systemic lupus erythematosus. Clinical presentation, histology, survival, and outcome. Medicine. 1997;76(3):192202. https://doi.org/10.1097/00005792-199705000-00005.

65. Suzuki A, Asazuma N, Kikuchi E, Kawanobe T, Horimoto Y, Yokobari R, et al. "Possible primary antiphospholipid syndrome" with concurrent diffuse alveolar hemorrhaging and Libman-sacks endocarditis mimicking catastrophic antiphospholipid syndrome. Intern Med. 2012;51(7):813-6. https://doi.org/10.2169/ internalmedicine.51.6592.

66. Scheiman Elazary A, Klahr PP, Hershko AY, Dranitzki Z, Rubinow A, Naparstek Y. Rituximab induces resolution of recurrent diffuse alveolar hemorrhage in a patient with primary antiphospholipid antibody syndrome. Lupus. 2012;21(4):438-40. https://doi.org/10. 1177/0961203311422713.

67. Bucciarelli S, Espinosa G, Cervera R, Erkan D, Gomez-Puerta JA, Ramos-Casals $M$, et al. Mortality in the catastrophic antiphospholipid syndrome: causes of death and prognostic factors in a series of 250 patients. Arthritis Rheum. 2006;54(8):2568-76. https://doi.org/10.1002/art.22018.

68. Helgeson SA, Heckman AJ, McCain JD, Cowart JB, Maniaci MJ, Garland JL. A 32-year-old man with hypoxemia and bilateral upper-lobe predominant ground-glass infiltrates on chest imaging. Oxf Med Case Reports. 2018;10(10):335-8. https://doi.org/10. 1093/omcr/omy072.

69.• Legault K, Schunemann H, Hillis C, Yeung C, Akl EA, Carrier M, et al. McMaster RARE-Bestpractices clinical practice guideline on diagnosis and management of the catastrophic antiphospholipid syndrome. J Thromb Haemost. 2018;16:1656-64. https://doi.org/ $10.1111 /$ jth.14192. Recent guidelines on the diagnosis and management of catastrophic APS

70. Isshiki T, Sugino K, Gocho K, Furuya K, Shimizu H, Sekiya M, et al. Primary antiphospholipid syndrome associated with diffuse alveolar hemorrhage and pulmonary thromboembolism. Intern Med. 2015;54(16):2029-33. https://doi.org/10.2169/ internalmedicine.54.4058.

71. Pazzola G, Zuily S, Erkan D. The challenge of bleeding in antiphospholipid antibody-positive patients. Curr Rheumatol Rep. 2015;17(2). https://doi.org/10.1007/s11926-014-0481-0.

72. Edwards MH, Pierangeli S, Liu X, Barker JH, Anderson G, Harris EN. Hydroxychloroquine reverses thrombogenic properties of antiphospholipid antibodies in mice. Circulation. 1997;96(12): 4380-4. https://doi.org/10.1161/01.Cir.96.12.4380.

73. Lopez-Pedrera C, Ruiz-Limon P, Aguirre MA, Barbarroja N, PerezSanchez C, Buendia P, et al. Global effects of fluvastatin on the prothrombotic status of patients with antiphospholipid syndrome. Ann Rheum Dis. 2011;70(4):675-82. https://doi.org/10.1136/ard. 2010.135525.

74. Ruiz-Irastorza G, Egurbide MV, Pijoan JI, Garmendia M, Villar I, Martinez-Berriotxoa A, et al. Effect of antimalarials on thrombosis and survival in patients with systemic lupus erythematosus. Lupus. 2006;15(9):577-83. https://doi.org/10.1177/0961203306071872.

75. Schmidt-Tanguy A, Voswinkel J, Henrion D, Subra JF, Loufrani L, Rohmer V, et al. Antithrombotic effects of hydroxychloroquine in primary antiphospholipid syndrome patients. J Thromb Haemost. 2013;11(10):1927-9. https://doi.org/10.1111/jth.12363.

Publisher's Note Springer Nature remains neutral with regard to jurisdictional claims in published maps and institutional affiliations. 